\title{
Diffusion tensor imaging predicts the outcome of constraint-induced movement therapy in chronic infarction patients with hemiplegia: A pilot study
}

\author{
Kohei Marumoto ${ }^{\mathrm{a}, *}$, Tetsuo Koyama ${ }^{\mathrm{b}}$, Masashi Hosomi ${ }^{\mathrm{a}}$, Takashi Takebayashic ${ }^{\mathrm{c}}$, Keisuke Hanada ${ }^{\mathrm{c}}$, \\ Sayaka Ikeda ${ }^{\mathrm{a}}$, Norihiko Kodama ${ }^{\mathrm{a}}$ and Kazuhisa Domen ${ }^{\mathrm{a}}$ \\ a Department of Physical Medicine and Rehabilitation, Hyogo College of Medicine, 1-1 Mukogawa-cho, \\ Nishinomiya, Hyogo, Japan \\ ${ }^{\mathrm{b}}$ Department of Rehabilitation Medicine, Nishinomiya Kyoritsu Neurosurgical Hospital, \\ 11-1 Imazu-Yamanaka-cho, Nishinomiya, Hyogo, Japan \\ ${ }^{\mathrm{c}}$ Department of Rehabilitation, The Hospital of Hyogo College of Medicine, 1-1 Mukogawa-cho, \\ Nishinomiya, Hyogo, Japan
}

\begin{abstract}
.
Purpose: To test whether diffusion tensor imaging could evaluate potential motor capability of patients with chronic cerebral infarction.

Methods: We used constraint-induced movement therapy (CIMT) as a rehabilitation, which reveals potential motor capability. We also investigated the relationship between the outcome of CIMT and the ratio between fractional anisotropy values (rFA) in affected and unaffected sites of the corticospinal tract before CIMT. Imaging was performed in cerebral infarction patients $(n=14)$ and the rFA of the posterior limb of internal capsule (PLIC) was measured before CIMT. Patients were evaluated before and after CIMT using the Fugl-Meyer (F-M) assessment, Wolf Motor Function Test, Action Research Arm Test, and Motor Activity Log and association between PLIC- rFA and these scores was determined.

Results: All patients showed an improvement in mobility following the 10-day CIMT session. Strong positive correlation was found only between F-M after CIMT and PLIC-rFA $(r=0.8098, p=0.0004)$. A strong linear relationship was observed after CIMT.

Conclusion: These data support PLIC-rFA as a new marker of the CIMT-induced improvement in motor function (F-M).
\end{abstract}

Keywords: Constraint-induced movement therapy, rehabilitation, stroke, diffusion tensor imaging, fractional anisotropy, corticospinal tract

\footnotetext{
*Corresponding author: Kohei Marumoto, Department of Physical Medicine and Rehabilitation, Hyogo College of Medicine, 1-1 Mukogawa-cho, Nishinomiya, Hyogo, Japan 663-8501. Tel.: +81 79845 6881; Fax: +81 79845 6948; E-mail: k.maru5081@ gmail.com
}

\section{Introduction}

Brain injury can cause a range of symptoms including motor paralysis, sensory impairment, and higher brain dysfunction depending on the site of injury. According to the classical concept, there is almost no 
recovery of brain function after brain damage, thus the main objective of rehabilitation has been the improvement of activities of daily living by means such as the development of compensatory movement (Dobkin, 2004). However, recent developments in brain science have revolutionized our understanding of approach to the rehabilitation of brain damage (Dobkin, 2008; Dobkin et al., 2012; Gauthier et al., 2008; Han et al., 2008; Kwakkel et al., 1999; Langhorne et al., 2009; Taub et al., 1994; Timmermanns et al., 2010).

Rehabilitation medicine is currently explored to improve motor function recovery in post-stroke patients. The most widely accepted treatment is constraint-induced movement therapy (CIMT), during which the non-paralyzed hand is immobilized while the exercise routines based on daily living activities are performed with the paralyzed hand (Wolf et al., 2006). This approach focusses on a rehabilitation treatments which reveal potential motor capability based on the concept that physical training can overcome the learned nonuse resulting from post-stroke hemiplegia (a phenomenon by which movement or behavior is suppressed because initial attempts to use the affected limb results in failure, causing the individual to suppress the movement and never realize the potential for recovery) (Taub et al., 2006). The ideal approach to monitor the progress of a patient during CIMT is to use a combination of neural imaging and functional assessments to determine whether the exercise routine and intensity lead to positive or negative neurogeneration in terms of mobility (Wittenberg \& Schaechter, 2009).

The quantitative methods for evaluating the level of damage to the cortex have also been developed. These include positron emission tomography and functional magnetic resonance imaging (Dong et al., 2006; Wittenberg et al., 2003). Functional magnetic resonance imaging and other functional brain imaging methods are often used when evaluating the potential for functional reconstruction. However, even if the brain activity suggests the potential for compensation or reconstruction, it is impossible to determine whether this is the most favorable activity for therapy based on functional magnetic resonance imaging alone (Kelly et al., 2006). Also, these techniques were inadequate to monitor the impact of rehabilitation therapy on brain structure. The latest paper reported that white matter integrity assessed by diffusion tensor imaging is a stronger predictor of motor function than functional magnetic resonance imaging parameter in patients with stroke (Qiu et al., 2011).
Diffusion tensor imaging evaluates the random motion of water molecules, and uses tensor analysis to obtain overall data on the direction and pattern of diffusion. The fractional anisotropy (FA) value, an indicator of anisotropy (directional dependence) is frequently used in quantitative evaluation (Pierpaoli et al., 1996). Associations between diffusion tensor imaging and post-stroke motor function, prediction of prognosis (Lindenberg et al., 2010), and higher brain dysfunction due to brain trauma (Kinnunen et al., 2011) have been described. Especially, numerous studies have also used diffusion tensor imaging to investigate the relationship between corticospinal tract integrity and upper extremity motor function or predict the prognosis for return of upper extremity function (Jang, 2010), but few have used it to measure the outcome of a rehabilitation. For those that have, results have been equivocal, and a consensus regarding its predictive power has not been reached (Sterr et al., 2010; Stinear et al., 2007). There is a published report that show successful prediction of rehabilitation outcome using diffusion tensor imaging. However, Stinear et al. (2007) used transcranial magnetic stimulation together with diffusion tensor imaging in this study. There are no published report that show successful prediction of CIMT outcome using diffusion tensor imaging alone.

The aim of this study was to test whether diffusion tensor imaging could evaluate potential motor capability of patients with chronic cerebral infarction. We used CIMT this time as a rehabilitation, which reveals potential motor capability. We also investigated whether there was an association between corticospinal tract integrity and restoration of upper extremity motor function to determine if diffusion tensor imaging could be used to predict treatment prognosis and inform treatment strategy.

\section{Methods}

\subsection{Subjects}

Subjects were patients with hemiparesis in the chronic stages of infarction ( $\geq 6$ months from onset) who were hospitalized at the Hyogo College of Medicine Hospital for CIMT between August 2010 and March 2013. All patients met the following higherfunctioning criteria as established by Winstein et al. (2003) and Wolf et al. (2006). Patients: 1) had suffered a stroke caused by a cerebral infarct or intracerebral 
hemorrhage; 2) had both interphalangeal and metacarpophalangeal joints that could be extended by at least $10^{\circ}$ and the wrist joints by at least $20^{\circ} ; 3$ ) had no severe balance or walking disorders, and were able to care for oneself independently (e.g., were able to eat, go to the bathroom, and walk without assistance); 4) exhibited no clear signs of dementia, mental disorder, or aphasia that presented an obstacle to daily living; 5) lacked of excessive pain, spasticity, ataxia, or frailty as determined by clinical judgment; 6) had no other severe end-stage or uncontrolled medical conditions. Each participant provided informed consent in writing, in advance, and the study was approved by the Institutional Review Board of the Hyogo College of Medicine Hospital.

\subsection{Protocol for constraint-induced movement therapy}

The training program was established according to Hosomi's procedure (Hosomi et al., 2011). The less affected upper extremity was constrained using a mitten or sling during training. Before the training was initiated, a physician or therapist assessed the functional impairment of the most affected upper extremity, and interviewed the patient on their ability to conduct daily activities (e.g., eating, grooming, or dressing). The nature of the therapy was tailored according to each individual. The CIMT training was carried out $5 \mathrm{~h}$ per day $(2 \mathrm{~h}$ in the morning, $1 \mathrm{~h}$ of rest, and $3 \mathrm{~h}$ in the afternoon) on 10 weekdays, in 2 consecutive weeks. Of the five hours of daily training, two (40\% of the training time) involved direct (one-on-one) therapist supervision. The remaining three $(60 \%$ of the training time) were designated for self-training. Our protocol constrains the less affected upper extremity only during training.

\subsection{Functional assessment methods}

We performed assessments of upper extremity function before and after the 10 weekdays of intervention. For assessments, we used tasks related to upper extremity function from each the Fugl-Meyer Assessment (F-M) (Fugl-Meyer et al., 1975), the Wolf Motor Function Test (Wolf et al., 2001), the Action Research Arm Test (Lang et al., 2006), and the Motor Activity Log (Uswatte et al., 2006). The F-M quantitatively evaluates the movement, sensation, and balance functions of a patient's extremities and trunk after a stroke. We used the upper extremity component of this examination, which evaluates function of the shoulder, elbow, forearm, wrist, and hand using a three-point ordinal score (where 0 indicates cannot perform; 1 indicates can partially perform; and 2 indicates can fully perform). The maximum score for the upper extremity component of F-M is 66. The Wolf Motor Function Test evaluates the function of the more affected upper extremity and consists of 15 movement items. There are two components: a temporal and qualitative assessment. A performance time is recorded for each movement. We assessed total time (second) of all 15 movement items. In addition, the Functional Ability Scale (the qualitative assessment) evaluates each movement task on a 6 -level scale of 0 to 5 points $(0=$ does not attempt to $5=$ normal movement). We assessed average score of 15 movement items. The maximum score for Wolf Motor Function Test (Functional Ability Scale) is 5.0. The Action Research Arm Test also assesses function of upper extremity using ordinal scoring on 19 items, where 0 indicates no movement and 3 indicates normal movement. Item scores are summed to create four subscale scores: gross motor (9-point maximum), grasp (18-point maximum), grip (12-point maximum), and pinch (18-point maximum), and a total scale score (57point maximum, indicating normal performance) (Koh et al., 2006). Lastly, the Motor Activity Log is an instrument that scores how much (Amount of Use scale) the patient's impaired arm was used to accomplish each of the 14 daily activities. This scale is anchored at six points $(0=$ never used, $5=$ same as pre-stroke $)$. We assessed average score of 14 activities of daily living. The maximum score for Motor Activity Log (Amount of Use) is 5.0. The evaluator who did not know the rehabilitation contents assessed the function of patients in our study.

\subsection{Diffusion tensor imaging acquisition}

Magnetic resonance imaging acquisition and analysis in this study were performed according to the methods described by Koyama et al. (2011). To determine if diffusion tensor imaging could be used to predict treatment prognosis, diffusion tensor imaging was performed using a 3-T Magnetic resonance imaging scanner (Trio; Siemens AG, Erlangen, Germany) with a 32-channel head coil at the Nishinomiya Kyoritsu Neurosurgical Hospital one week 
before CIMT. Using a single-shot echo-planar imaging sequence, the diffusion tensor imaging scheme included acquisition of 12 images with noncollinear diffusion gradients $\left(b=1000 \mathrm{~s} / \mathrm{mm}^{2}\right)$ and 1 nondiffusion-weighted image $\left(b=0 \mathrm{~s} / \mathrm{mm}^{2}\right)$. A total of 64 axial slices was obtained for each patient. The field of view was $230.4 \times 230.4 \mathrm{~mm}^{2}$, the acquisition matrix was 128 , and the slice thickness was $3 \mathrm{~mm}$ without a gap, resulting in voxel dimensions of $1.8 \mathrm{~mm} \times 1.8 \mathrm{~mm} \times 3.0 \mathrm{~mm}$. The echo time was $83 \mathrm{~ms}$, and the repetition time was $7000 \mathrm{~ms}$. Along with the diffusion tensor imaging scan, T1-weighted and T2weighted Magnetic resonance imaging were obtained for other diagnostic uses. Including those scans, the total time for Magnetic resonance imaging acquisition was approximately $20 \mathrm{~min}$ per patient.

\subsection{Image processing}

The brain image analysis package FMRIB Software Library (FSL), comprising of various tools, including a Brain Extraction Tool, FMRIB's Diffusion Toolbox, FMRIB's Nonlinear Image Registration Tool, and FSLVIEW, was used for image processing (Analysis Group, 2010). Using the FDT tool to align all images in volumetric relation to the first image $\left(b=0 \mathrm{~s} / \mathrm{mm}^{2}\right)$, diffusion tensor imaging data were corrected for motion and eddy current distortion. Extracerebral matter was excluded from the images using the BET tool; then, to evaluate tensor diffusion and calculate each patient's brain FA values, diffusion tensor imaging data were analyzed using the FDT tool (FA brain map). Using FMRIB's Nonlinear Image Registration Tool, these FA values were mapped to a standard stereotaxic space (International Consortium of Brain Mapping DTI81 Atlas: ICBM DTI-81 Atlas) (Laboratory of Brain Anatomical MRI. Human White Matter Anatomy and Development, 2010; Mori et al., 2008). As recommended in the manual, we employed "standard tasks" settings for FMRIB's Nonlinear Image Registration Tool. Spatial transformations of the FA brain maps were confirmed by visual comparisons with images generated by FSLVIEW. When using FSLVIEW, we visually confirmed that the regions of interest were within the white matter in all subjects.

\subsection{Regions of interest and Lesion size}

Consistent with previous studies (Qiu et al., 2011; Sterr et al., 2010), we specified Regions of interest in

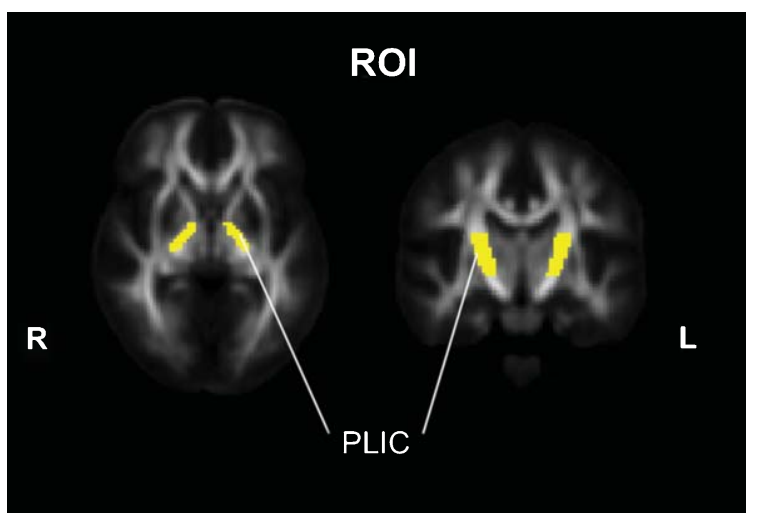

Fig. 1. Region of interest (ROI) within the posterior limb of internal capsule (PLIC). The ROI was positioned using the International Consortium of Brain Mapping DTI-81 Atlas (ICBM DTI-81 Atlas).

the posterior limb of internal capsule (PLIC). To do this, we used templates for the Region of interest as defined in the ICBM DTI-81 Atlas (Fig. 1). FA values were assessed using FSL UTILS, and mean values for single voxels were then estimated. In subsequent analyses, the ratio between FA values (rFA) in affected and unaffected sites of the posterior limb of internal capsule (PLIC-rFA) were calculated (Koyama et al., 2011). Infarction size was identified and manually measured under supervision of neurologist. To calculate irregular volumes, we used the following formula: $\mathrm{A} \times \mathrm{B} \times \mathrm{C} / 2$. We measured the largest diameter (A) and the perpendicular diameter (B) of the slices where the lesion was visible (Pantano et al., 1999). FA values and infarction size were double-blind to the patient and therapist during evaluation and medical treatment.

\subsection{Statistical analyses}

All statistical analyses were performed using the JMP software package (SAS Institute, Cary, NC). For analyses evaluating efficacy, the intervention data were compared using paired t - test. We used Spearman's rank correlation coefficient to identify possible associations between both rFA (or lesion size) and the clinical assessment (F-M, Wolf Motor Function Test (performance time), Wolf Motor Function Test (Functional Ability Scale), Action Research Arm Test, and Motor Activity Log (Amount of Use)) scores before and after the intervention. A simple regression analysis was performed to determine liner relationship. Statistical significance was defined as $P<0.05$. 


\section{Results}

The 14 post-stroke right handed patients without somatosensory and visual deficit included in this study (mean age 55 years) were mostly males $(85 \%)$ with structural damage to the left hemisphere (64\%), only subcortical lesions $(71 \%)$, and a mean PLIC-rFA of 0.92 (Table 1). Brain magnetic resonance imaging of each patient captured prior to CIMT (Fig. 2). Therefore, most patients experienced a reduction in mobility of the right limbs. All patients initially presented a significant loss of mobility based on at least four of the five parameters, with a mean F-M of 46 over 66 (range 38-58), mean Wolf Motor Function Test (performance time) of 210 seconds (range 41-648), mean Wolf Motor Function Test (Functional Ability Scale) of 3.3 over 5.0 (range 2.4-5.0), mean Action Research Arm Test of 39 over 57 (range 22-56), and mean Motor Activity Log (Amount of Use) 1.73 over 5.0 (range 0.64-3.35) (Table 2). While Patient \#14 performed almost normally based on F-M, Patient \#7 appeared healthy based on Action Research Arm Test. This variability is expected because of the different types of impairment, and the different focus of each test. This type of analysis supports the need to conduct several tests to assess the status of each patient within a heterogeneous group.

The CIMT session significantly improved the mobility of the post-stroke patients (Table 2). With respect to F-M, all patients showed good score after CIMT, $(p<0.0001$, Fig. 3A), likewise, Wolf
Motor Function Test (performance time) $(p=0.0082$, Fig. 3B), Wolf Motor Function Test (Functional Ability Scale) $(p=0.0012$, Fig. 3C), Action Research Arm Test $(p<0.0001$, Fig. 3D), and Motor Activity Log (Amount of Use) ( $p=0.0001$, Fig. 3E). The improvement in each score was higher for the type of disability that was best documented by each test. These data demonstrate that each patient responded to the CIMT by significant improvement in their specific disability, as shown by the appropriate assessment methods. Correlation analyses conducted between lesion size and the functional assessment data both pre-CIMT and post-CIMT revealed no significant relationship. Moreover, there was no relationship between lesion size and functional gains.

Strong positive correlation was found only between F-M after CIMT and PLIC-rFA ( $r=0.8098$, $p=0.0004$ ) (Fig. 4). Also, there was no relationship between PLIC-rFA and functional gains. FA is the better prediction of rehabilitation outcome (post - CIMT) than the lesion size.

\section{Discussion}

\subsection{Predict the outcome of rehabilitation}

Rehabilitation medicine is currently used to improve therapeutic approaches to stroke-induced motor paralysis. However, the imaging techniques proposed to monitor changes in brain neural network during

Table 1

Participant characteristics

\begin{tabular}{|c|c|c|c|c|c|c|c|}
\hline Patient & Sex & Age & $\begin{array}{c}\text { Time since } \\
\text { stroke (Month) }\end{array}$ & $\begin{array}{c}\text { Affected } \\
\text { hemisphere }\end{array}$ & $\begin{array}{c}\text { Affected } \\
\text { structures }\end{array}$ & $\begin{array}{c}\text { Lesion } \\
\text { size }\left(\mathrm{cm}^{3}\right)\end{array}$ & PLIC-rFA \\
\hline 1 & M & 37 & 39 & $\mathrm{~L}$ & $\mathrm{CR}$ & 3.75 & 0.86 \\
\hline 2 & M & 66 & 16 & $\mathrm{R}$ & CR & 3.45 & 0.871 \\
\hline 3 & $\mathrm{M}$ & 62 & 26 & $\mathrm{~L}$ & PLIC & 7.5 & 0.879 \\
\hline 4 & $\mathrm{M}$ & 66 & 16 & $\mathrm{R}$ & PLIC & 3.75 & 0.888 \\
\hline 5 & $\mathrm{M}$ & 67 & 52 & $\mathrm{R}$ & GP, Put, PLIC & 9.00 & 0.904 \\
\hline 6 & $\mathrm{M}$ & 74 & 35 & $\mathrm{~L}$ & PLIC & 1.13 & 0.907 \\
\hline 7 & $\mathrm{M}$ & 62 & 11 & $\mathrm{R}$ & CR, GP, Put & 3.75 & 0.919 \\
\hline 8 & $\mathrm{M}$ & 55 & 38 & $\mathrm{~L}$ & CR, Put, Motor Cx & 7.50 & 0.932 \\
\hline 9 & $\mathrm{~F}$ & 65 & 12 & $\mathrm{~L}$ & $\mathrm{CR}$ & 1.20 & 0.932 \\
\hline 10 & $\mathrm{M}$ & 37 & 9 & $\mathrm{~L}$ & CR, Motor Cx & 15.0 & 0.932 \\
\hline 11 & $\mathrm{~F}$ & 21 & 144 & $\mathrm{R}$ & CR, GP, Put, Insular & 86.4 & 0.945 \\
\hline 12 & $\mathrm{M}$ & 65 & 44 & $\mathrm{~L}$ & $\mathrm{CR}$ & 7.5 & 0.946 \\
\hline 13 & $\mathrm{M}$ & 42 & 25 & $\mathrm{~L}$ & GP, Put, PLIC & 6.0 & 0.967 \\
\hline 14 & M & 60 & 33 & $\mathrm{~L}$ & Motor Cx & 18.0 & 1.001 \\
\hline
\end{tabular}

Abbreviations: PLIC, Posterior limb of internal capsule; rFA, the ratios between fractional anisotropy values in the affected and unaffected sites; CR, corona radiata; GP, globus pallidus; Put, putamen; Cx, cortex. 


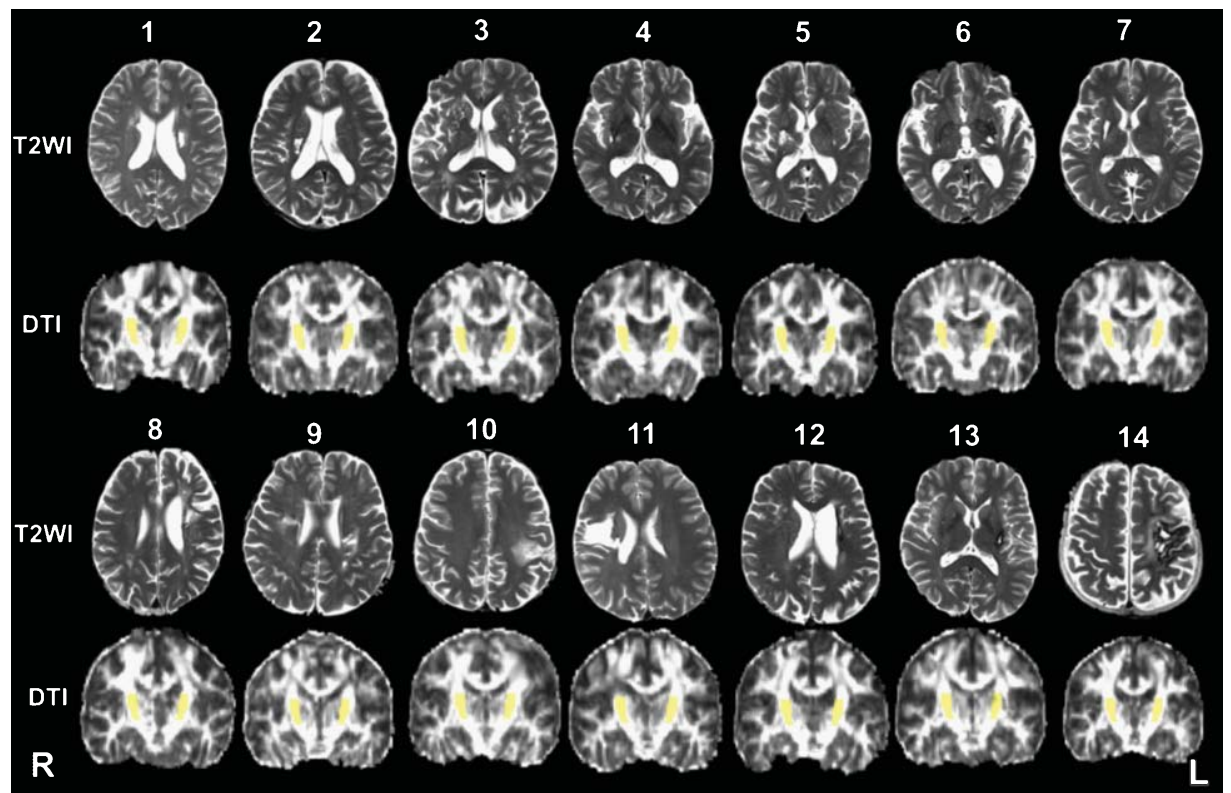

Fig. 2. Brain magnetic resonance imaging of each patient captured prior to constraint-induced movement therapy (CIMT). Each pair corresponds to the T2-weighted image (T2WI) and diffusion tensor image (DTI) of each patient, arranged in ascending order of the ratios between FA values $(\mathrm{rFA})$ in the affected and unaffected regions of interest within the posterior limb of the internal capsule (PLIC) $(1$ to $14=$ lowest to highest).

Table 2

Assessments of upper extremity function before and after CI therapy

\begin{tabular}{|c|c|c|c|c|c|c|c|c|c|c|}
\hline & \multicolumn{2}{|c|}{$\begin{array}{c}\text { F-M } \\
\text { (total score) }\end{array}$} & \multicolumn{2}{|c|}{$\begin{array}{l}\text { WMFT-Time } \\
(\mathrm{sec})\end{array}$} & \multicolumn{2}{|c|}{$\begin{array}{l}\text { WMFT-FAS } \\
\text { (average score) }\end{array}$} & \multicolumn{2}{|c|}{$\begin{array}{c}\text { ARAT } \\
\text { (total score) }\end{array}$} & \multicolumn{2}{|c|}{$\begin{array}{c}\text { MAL-AOU } \\
\text { (average score) }\end{array}$} \\
\hline & pre & post & pre & post & pre & post & pre & post & pre & post \\
\hline 1 & 38 & 49 & 648 & 192 & 3.2 & 3.8 & 22 & 28 & 0.71 & 2.43 \\
\hline 2 & 42 & 49 & 52 & 42 & 3.3 & 3.6 & 34 & 55 & 1.37 & 2.62 \\
\hline 3 & 48 & 49 & 88 & 38 & 2.4 & 3.6 & 54 & 55 & 2.42 & 3.42 \\
\hline 4 & 48 & 50 & 204 & 49 & 3.0 & 3.8 & 42 & 56 & 1.20 & 1.80 \\
\hline 5 & 48 & 52 & 463 & 221 & 2.8 & 3.9 & 34 & 49 & 1.83 & 2.45 \\
\hline 6 & 48 & 50 & 57 & 40 & 3.6 & 3.8 & 53 & 56 & 1.85 & 2.64 \\
\hline 7 & 45 & 51 & 41 & 37 & 3.3 & 3.3 & 56 & 57 & 1.36 & 2.08 \\
\hline 8 & 41 & 48 & 205 & 232 & 3.2 & 3.1 & 34 & 40 & 1.35 & 2.00 \\
\hline 9 & 43 & 52 & 211 & 61 & 3.4 & 3.6 & 36 & 48 & 0.64 & 2.85 \\
\hline 10 & 45 & 54 & 453 & 97 & 3.6 & 4.0 & 24 & 32 & 1.07 & 2.35 \\
\hline 11 & 52 & 57 & 285 & 200 & 3.0 & 3.6 & 33 & 42 & 1.71 & 2.00 \\
\hline 12 & 50 & 57 & 97 & 21 & 3.0 & 3.5 & 47 & 55 & 3.32 & 3.42 \\
\hline 13 & 45 & 58 & 77 & 19 & 5.0 & 5.0 & 47 & 57 & 3.35 & 4.14 \\
\hline 14 & 58 & 59 & 66 & 37 & 4.1 & 4.4 & 40 & 46 & 2.07 & 2.14 \\
\hline Mean & 46 & 52 & 210 & 91 & 3.3 & 3.7 & 39 & 48 & 1.73 & 2.59 \\
\hline (SEM) & (1.3) & $(1.0)$ & (50) & (21) & $(0.1)$ & $(0.1)$ & $(2.8)$ & $(2.5)$ & $(0.2)$ & $(0.1)$ \\
\hline
\end{tabular}

Abbreviations: F-M, Fugl-Meyer Assessment (upper extremity component); WMFT-Time, Wolf Motor Function Test (performance time); WMFT-FAS, Wolf Motor Function Test (Functional Ability Scale); ARAT, Action Research Arm Test; MAL-AOU, Motor Activity Log (Amount of Use); SEM, Standard Errors of the Mean.

therapy suffer from a lack of resolution and sensitivity (Wittenberg \& Schaechter, 2009), which may only be able to be accomplished by the analysis of multifaceted diffusion tensor imaging. Current applications for the resulting diffusion tensor imaging parameter include intensive rehabilitation of the affected upper extremity (Lindenberg et al., 2012; Stinear et al., 2007). Therefore, the aim of the present study was to test whether PLIC-rFA may be used to evaluate potential motor capability in the case of chronic cerebral infarction. 
A
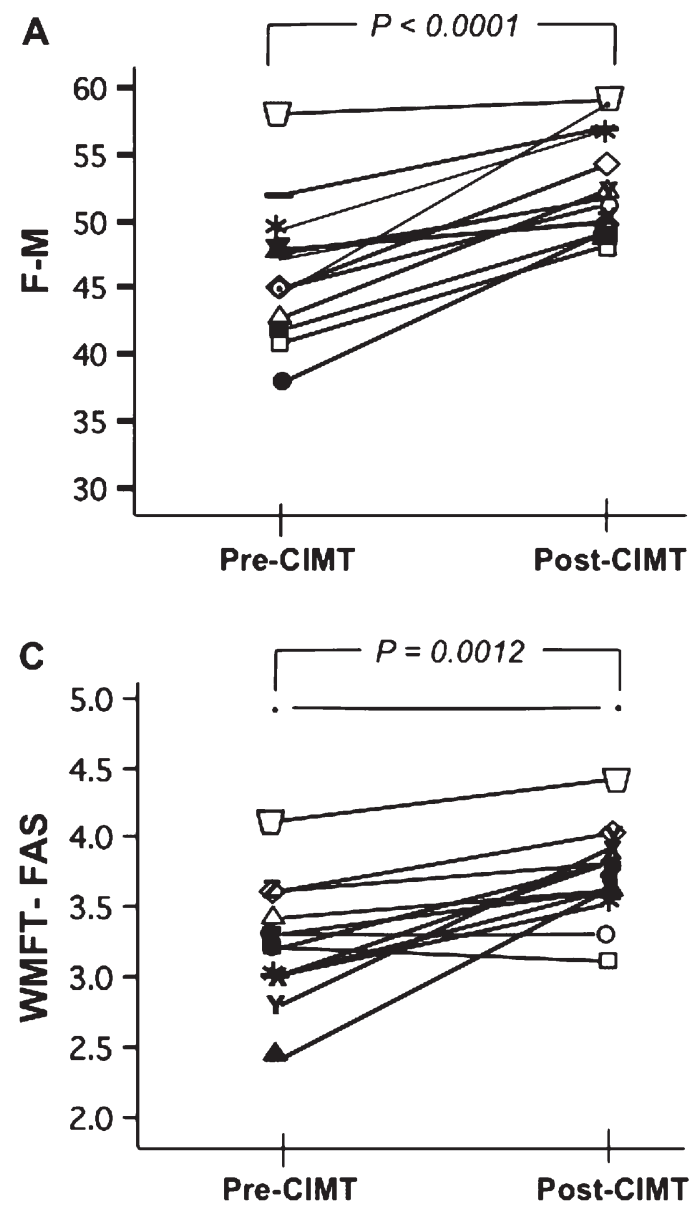

E

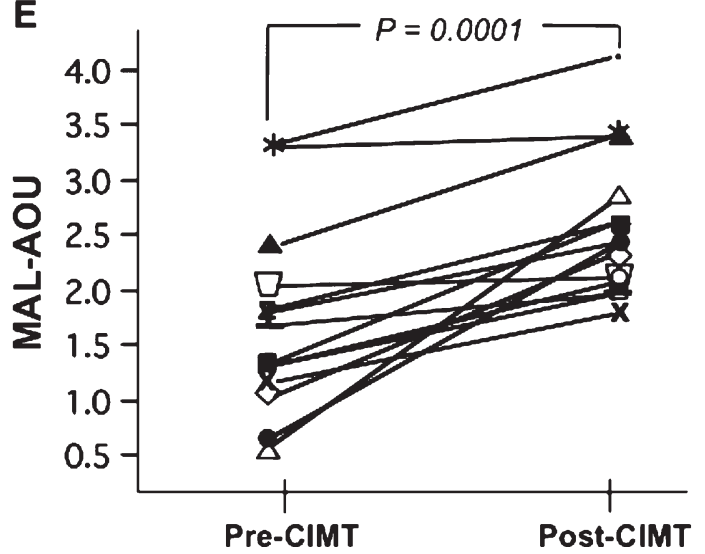

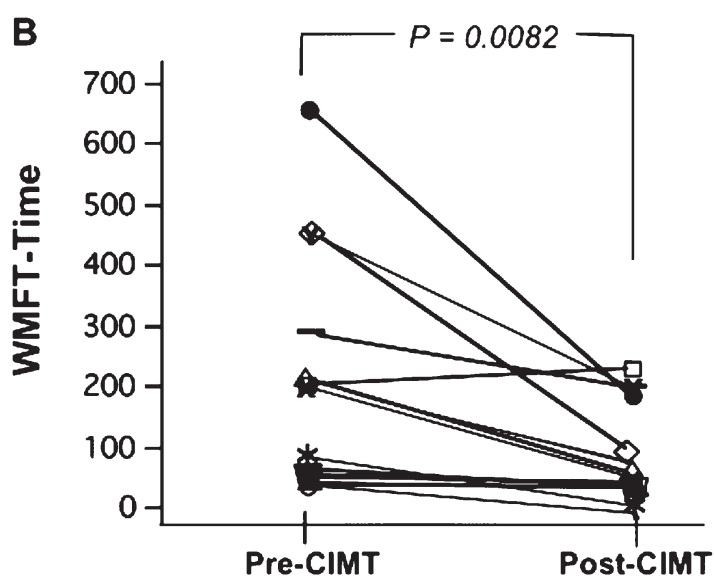
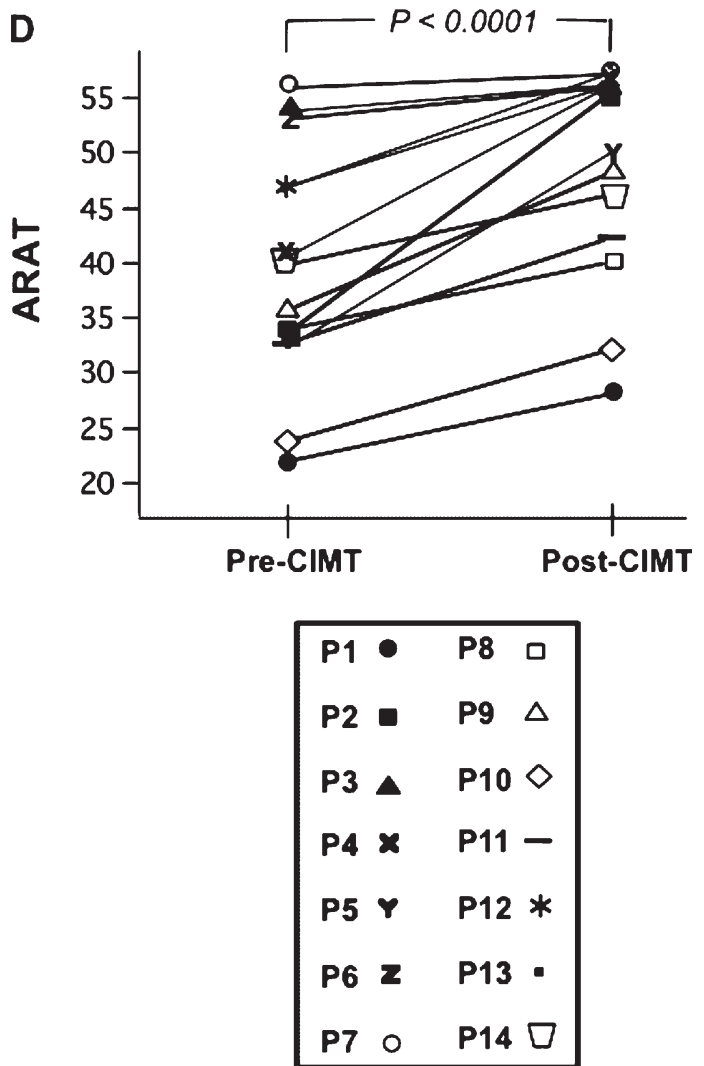

Fig. 3. (A-E). Comparing the Functional assessment data before and after CIMT. The CIMT session significantly improved the mobility of the post-stroke patients. With respect to F-M, all patients showed good score after CIMT, $(p<0.0001$, Fig. 3A), likewise, WMFT-Time $(p=0.0082$, Fig. 3B), WMFT-FAS ( $p=0.0012$, Fig. 3C), ARAT ( $p<0.0001$, Fig. 3D) and MAL-AOU ( $p=0.0001$, Fig. 3E). Abbreviations: CIMT, constraintinduced movement therapy; F-M, Fugl-Meyer Assessment (upper extremity component); WMFT-Time, Wolf Motor Function Test (performance time); WMFT-FAS, Wolf Motor Function Test (Functional Ability Scale); ARAT, Action Research Arm Test; MAL-AOU, Motor Activity Log (Amount of Use). 


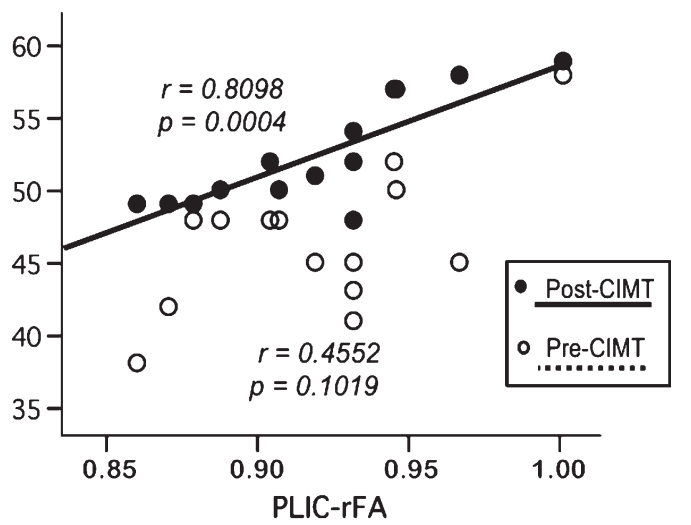

$\{$ F-M $\}$ post $=82.4$ \{PLIC-rFA $\}-23.4$

Fig. 4. Correlation between PLIC-rFA and F-M upper extremity assessment before and after CIMT. Strong positive correlation was found only between F-M after CIMT and PLIC-rFA $(r=0.8098$, $p=0.0004)$. Regression analysis revealed that F-M was the only functional assessment that correlated positively with PLIC-rFA. An equation of the correlation between PLIC-rFA and F-M scores after CIMT was showed at the bottom. Abbreviations: PLIC, Posterior limb of internal capsule; rFA, the ratios between fractional anisotropy values in the affected and unaffected sites; CIMT, constraint-induced movement therapy; F-M, Fugl-Meyer Assessment (upper extremity component).

We provide evidence that this diffusion tensor imaging index of corticospinal tract integrity correlates with the motor functions of these patients based on the F-M score system.

\subsection{Comparing the functional assessment data}

This study demonstrated that each patient responded to the CIMT by significant improvement in their specific disability, as shown by the appropriate assessment methods. Post-stroke patients exhibit a large variability in the affected brain areas, which result in various types of motor impairments. Since each functional assessment test measures different parameters, the progress of each patient will be best evaluated by certain tests, and not by the others, which explains the variability in responses between the tests for the same patient. Accordingly, the strongest response in all functional tests should be used to determine the percent improvement in motor function caused by CIMT. Therefore, motility assessment must be conducted with multiple tests to ensure that the progress of each post-stroke patient is properly documented during CIMT.

\subsection{Correlation analyses between PLIC-rFA and all the functional tests}

In the study, all patients showed an improvement in mobility following CIMT. Strong positive correlation was found only between F-M after CIMT and PLICrFA $(r=0.8098, p=0.0004)$. Stinear et al. (2007) used transcranial magnetic stimulation to evaluate the functional integrity of the corticospinal tract and diffusion tensor imaging to evaluate its structural integrity, and implemented a 30-day program of motor practice of the upper extremity. That study not only found an association between the asymmetry in FA and baseline F-M upper extremity score but also with the magnitude of the change in F-M upper extremity score before and after rehabilitation (after scores minus before scores). Sterr et al. (2010) used diffusion tensor imaging to produce an FA map and calculate the area of overlap between areas with decreased FA value and the corticospinal tract. They found that, although the area of overlap was correlated with Wolf Motor Function Test (performance time) and Wolf Motor Function Test (Functional Ability Scale) before CIMT, there was no association between the area of overlap and the magnitude of the change before and after CIMT. Globas et al. (2011) found that there was a tendency for rFA of the corticospinal tract to be associated with baseline F-M and Wolf Motor Function Test (performance time) but no association with treatment outcomes (difference between before and after) in either patients who underwent rehabilitation of both arms or those who underwent rehabilitation of the paralyzed side.

Previous research has failed to find a consistent relationship between corticospinal tract integrity and the outcome of rehabilitation, and few studies have predicted the outcome of rehabilitation. The reason may be that the amount of effects of rehabilitation commonly used to measure the outcome is the change in F-Mupper extremity or Wolf Motor Function Test scores (score after rehabilitation minus the score before rehabilitation). This method is susceptible to the influence of ceiling and floor effects, which may explain why the relationship between the outcome of rehabilitation and corticospinal tract integrity has been difficult to elucidate.

We hypothesize that there are biological limitations to post-stroke upper extremity dysfunction, and that this is restricted to some extent by the corticospinal tract. The degree to which residual corticospinal tract 
function can be elicited in the process of functional recovery is an extremely important problem with respect to post-stroke upper extremity paralysis. However, the learned nonuse may be one possible cause of the gap that can occur between this biological limitation (residual corticospinal tract function) and actual function. For this reason, patients may approach the biological limit of functional recovery following CIMT, since this therapy involves overcoming the learned nonuse. Consistent with this hypothesis, the variation in the relationship between F-M upper extremity function and PLIC-rFA, observed before CIMT, disappeared following CIMT, and a strong linear relationship was observed between the two measures (Fig. 4). This relationship suggests that PLIC-rFA can be used to predict the outcome of CIMT on upper extremity function (F-M upper extremity score).

\subsection{Limitation and future study}

Unfortunately, this study has limitations. A limitation of this study was that the relationship between F-M and PLIC-rFA might not be the specific relationship brought about by CIMT. Namely, PLIC-rFA might reflect a potential motor capability if the patients properly received rehabilitation, whatever it is. Second limitation was its small sample size $(n=14)$ and variety of stroke lesions (including cortical and subcortical lesions). Patients with cortical lesions and patients with subcortical lesions should be discussed separately since motor cortical lesion as well as the integrity of the descending motor pathways may affect the outcome. However, we cannot discuss adequately this point because its sample size is small. The relationship between F-M upper extremity function and PLIC-rFA must be validated by similar studies involving larger numbers of cases. Third limitation is that we did not evaluate the long-term effect of CIMT. Longterm functional improvement after CIMT may not be dependent on the corticospinal tract, and it is possible that the effects of factors such as use-dependent plasticity (Ward et al., 2006) in multiple areas of the brain may enable recovery that exceeds biological limitations. This should be considered a priority for future research, since the pursuit of recovery which exceeds biological limitations is the primary goal of neurorehabilitation. Last limitation was that these patients in our study were not severely impaired. About more serious cases, the further research is needed.

\section{Conclusion}

Diffusion tensor imaging is a useful tool for prediction of improvement in motor function in chronic cerebral infarction patients undergoing CIMT.

\section{Declaration of conflicting interests}

The authors have no personal financial or institutional interest in any of the drugs, materials, or devices described in this article.

\section{Acknowledgments}

Part of this research was supported by Grant-inAid for Scientific Research (B), Japan Society for the Promotion of Science (KAKENHI [23300203]).

\section{References}

Analysis Group. FMRIB Software Library. 2010. [cited 2010. 28 October]; Available from: http://www.fmrib.ox.ac.uk/fsl/

Dobkin, B.H. (2004). Strategies for stroke rehabilitation. Lancet Neurol, 3(9), 528-536.

Dobkin, B.H. (2008). Training and exercise to drive poststroke recovery. Nat Clin Pract Neurol, 4(2), 76-85.

Dobkin, B.H., Plummer-D’Amato, P., Elashoff, R. \& Lee, J. (2010). International randomized clinical trial, stroke inpatient rehabilitation with reinforcement of walking speed (SIRROWS), improves outcomes. Neurorehabil Neural Repair, 24(3), 235242.

Dong, Y., Dobkin, B.H., Cen, S.Y., Wu, A.D. \& Winstein, C.J. (2006). Motor cortex activation during treatment may predict therapeutic gains in paretic hand function after stroke. Stroke, 37(6), 1552-1555.

Fugl-Meyer, A.R., Jaasko, L., Leyman, I., Olsson, S. \& Steglind, S. (1975). The post-stroke hemiplegic patient. 1. a method for evaluation of physical performance. Scand J Rehabil Med, 7(1), 13-31.

Gauthier, L.V., Taub, E., Perkins, C., Ortmann, M., Mark, V.W. \& Uswatte, G. (2008). Remodeling the brain: Plastic structural brain changes produced by different motor therapies after stroke. Stroke, 39(5), 1520-1525.

Globas, C., Lam, J.M., Zhang, W., et al. (2011). Mesencephalic corticospinal atrophy predicts baseline deficit but not response to unilateral or bilateral arm training in chronic stroke. Neurorehabil Neural Repair, 25(1), 81-87.

Han, C.E., Arbib, M.A. \& Schweighofer, N. (2008). Stroke rehabilitation reaches a threshold. PLoS Comput Biol, 4:e1000133.

Hosomi, M., Koyama, T., Takebayashi, T., et al. (2011). A Modified Method for Constraint-induced Movement Therapy: A 
Supervised Self-training Protocol. J Stroke Cerebrovasc Dis, doi:10.1016/j.jstrokecerebrovasdis.2011.04.004

Jang, S.H. (2010). Prediction of motor outcome for hemiparetic stroke patients using diffusion tensor imaging: A review. Neuro Rehabilitation, 27(4), 367-372.

Kelly, C., Foxe, J.J. \& Garavan, H. (2006). Patterns of normal human brain plasticity after practice and their implications for neurorehabilitation. Arch Phys Med Rehabil, 87(12 Suppl 2), S20-S29.

Kinnunen, K.M., Greenwood, R., Powell, J.H., et al. (2011). White matter damage and cognitive impairment after traumatic brain injury. Brain, 134(Pt 2), 449-463.

Koh, C.L., Hsueh, I.P., Wang, W.C., et al. (2006). Validation of the action research arm test using item response theory in patients after stroke. J Rehabil Med, 38(6), 375-380.

Koyama, T., Tsuji, M., Miyake, H., Ohmura, T. \& Domen, K. (2011). Motor Outcome for Patients with Acute Intracerebral Hemorrhage Predicted Using Diffusion Tensor Imaging: An Application of Ordinal Logistic Modeling. J Stroke Cerebrovasc Dis, 10.1016/j.jstrokecerebrovasdis.2011.03.004

Koyama, T., Tsuji, M., Nishimura, H., Miyake, H., Ohmura T. \& Domen, K. (2011). Diffusion tensor imaging for intracerebral hemorrhage outcome prediction: Comparison using data from the corona radiata/internal capsule and the cerebral peduncle. J Stroke Cerebrovasc Dis, doi:10.1016/ j.jstrokecerebrovasdis.2011.06.014

Kwakkel, G., Wagenaar, R.C., Twisk, J.W., Lankhorst, G.J. \& Koetsier, J.C. (1999). Intensity of leg and arm training after primary middle-cerebral-artery stroke: A randomised trial. Lancet, 354(9174), 191-196.

Laboratory of Brain Anatomical MRI. Human White Matter Anatomy and Development (2010). [cited 201028 October] Available from: http://cmrm.med.jhmi.edu/

Lang, C.E., Wagner, J.M., Dromerick, A.W. \& Edwards, D.F. (2006). Measurement of upper-extremity function early after stroke: Properties of the action research arm test. Arch Phys Med Rehabil, 87(12), 1605-1610.

Langhorne, P., Coupar, F. \& Pollock, A. (2009). Motor recovery after stroke: A systematic review. Lancet Neurol, 8(8), 741-754.

Lindenberg, R., Renga, V., Zhu, L.L., Betzler, F., Alsop, D. \& Schlaug, G. (2010). Structural integrity of corticospinal motor fibers predicts motor impairment in chronic stroke. Neurology, 74(4), 280-287.

Lindenberg, R., Zhu, L.L., Ruber, T. \& Schlaug, G. (2012). Predicting functional motor potential in chronic stroke patients using diffusion tensor imaging. Hum Brain Mapp, 33(5), 1040-1051.

Mori, S., Oishi, K., Jiang, H., et al. (2008). Stereotaxic white matter atlas based on diffusion tensor imaging in an ICBM template. Neuroimage, 40(2), 570-582.

Pantano, P., Caramia, F., Bozzao, L., Dieler, C. \& von Kummer, R. (1999). Delayed increase in infarct volume after cerebral ischemia: Correlations with thrombolytic treatment and clinical outcome. Stroke, 30(3), 502-507.
Pierpaoli, C., Jezzard, P., Basser, P.J., Barnett, A. \& Di Chiro, G (1996). Diffusion tensor MR imaging of the human brain. Radiology, 201(3), 637-648.

Qiu, M., Darling, W.G., Morecraft, R.J., Ni, C.C., Rajendra, J. \& Butler, A.J. (2011). White matter integrity is a stronger predictor of motor function than BOLD response in patients with stroke. Neurorehabil Neural Repair, 25(3), 275-284.

Sterr, A., Shen, S., Szameitat, A.J. \& Herron, K.A. (2010). The role of corticospinal tract damage in chronic motor recovery and neurorehabilitation: A pilot study. Neurorehabil Neural Repair, 24(5), 413-419.

Stinear, C.M., Barber, P.A., Smale, P.R., Coxon, J.P., Fleming, M.K. \& Byblow, W.D. (2007). Functional potential in chronic stroke patients depends on corticospinal tract integrity. Brain, $130(\mathrm{Pt}$ 1), 170-180.

Taub, E., Crago, J.E., Burgio, L.D., et al. (1994). An operant approach to rehabilitation medicine: Overcoming learned nonuse by shaping. J Exp Anal Behav, 61(2), 281-293.

Taub, E., Uswatte, G., Mark, V.W. \& Morris, D.M. (2006). The learned nonuse phenomenon: Implications for rehabilitation. Eura Medicophys, 42(3), 241-256.

Timmermans, A.A., Spooren, A.I., Kingma, H. \& Seelen, H.A. (2011). Influence of task-oriented training content on skilled arm-hand performance in stroke: A systematic review. $\mathrm{Neu}$ rorehabil Neural Repair, 24(9), 858-870.

Uswatte, G., Taub, E., Morris, D., Light, K. \& Thompson, P.A. (2006). The Motor Activity Log-28: Assessing daily use of the hemiparetic arm after stroke. Neurology, 67(7), 1189-1194.

Winstein, C.J., Miller, J.P., Blanton, S., Taub, E., Uswatte, G., Morris, D., et al. (2003). Methods for a multisite randomized trial to investigate the effect of constraint-induced movement therapy in improving upper extremity function among adults recovering from a cerebrovascular stroke. Neurorehabil Neural Repair, 17(3), 137-152.

Wittenberg, G.F., Chen, R., Ishii, K., Bushara, K.O., Eckloff, S., Croarkin, E., et al. (2003). Constraint-induced therapy in stroke: Magnetic-stimulation motor maps and cerebral activation. Neurorehabil Neural Repair, 17(1), 48-57.

Wittenberg, G.F. \& Schaechter, J.D. (2009). The neural basis of constraint-induced movement therapy. Curr Opin Neurol, 22(6), 582-588

Ward, N.S., Newton, J.M., Swayne, O.B., et al. (2006). Motor system activation after subcortical stroke depends on corticospinal system integrity. Brain, 129(Pt 3), 809-819.

Wolf, S.L., Catlin, P.A., Ellis, M., Archer, A.L., Morgan, B. \& Piacentino, A. (2001). Assessing Wolf motor function test as outcome measure for research in patients after stroke. Stroke, 32(7), 1635-1639.

Wolf, S.L., Winstein, C.J., Miller, J.P., et al. (2006). Effect of constraint-induced movement therapy on upper extremity function 3 to 9 months after stroke: The EXCITE randomized clinical trial. JAMA, 296(17), 2095-2104 